\title{
ÉTUDE DE L'APPRÉCIATION DE LA QUALITÉ DES CASÉINES LACTIQUES
}

\author{
par \\ JEAN PIEN \\ Ingénieur chimiste I. C. R. \\ Docteur ès sciences \\ Directeur des laboratoires \\ des "Fermiers Réunis" \\ Maro BERGIER \\ Ingénieur (I. A. N.) \\ du laboratoire des caséines \\ des "Fermiers Réunis "
}

Quand on étudie parallèlement la composition chimique des caséines lactiques et leur mode de comportement dans certaines applications industrielles (fabrication des colles, du papier couché, etc.), on est frappé de rencontrer assez souvent des anomalies telles que les suivantes : des caséines d'égale composition chimique donnant des résultats très différents dans la pratique et des caséines de bonne composition donnant de mauvais résultats (chutes de rendement, variations de viscosité et d'adhésivité, mousses, etc.).

Comme il ne saurait y avoir de doute sur la valeur de l'« observation industrielle » de la qualité des produits, on peut conclure que c'est "l'observation chimique " de la qualité des caséines qui, dans un certain nombre de cas, laisse à désirer. Autrement dit, l'analyse chimique des caséines telle qu'on l'exécute d'ordinaire (dosage des principaux éléments ou groupes d'éléments totaux) renseigne insuffisamment pour l'application à certaines industries et n'explique pas tous les accidents et toutes les anomalies rencontrées.

Cette remarque d'ordre général n'est pas inspirée de considérations théoriques. Elle nous a été fournie par la pratique. Nous avons l'occasion d'examiner en détail plus de mille caséines lactiques par an et de connaître les répercussions de l'application industrielle de oes produits. C'est ainsi que nous avons été amenés à concevoir l'insuffisance des données de l'analyse chimique ordinaire en présence d'anomalies imprévues et cependant indiscutables. Le problème que nous posons existe done et il existe pour tous ceux qui s'occupent de caséines (production, contrôle, utilisation). Il est inutile d'insister sur l'intérêt qui s'attache à la résolution d'un problème de cette importance.

$$
\text { ** * }
$$

Après avoir posé le problème, il s'agit de le définir.

Nous disons que l'analyse chimique ordinaire se révèle quelquefois insuffisante. De quelle analyse parlons-nous?

Les éléments que nous dosons dans une caséine lactique, sont les suivants :

$1^{0}$ Humidité (à poids minimum à $100^{\circ}$ ). 
20 Matières minérales par la méthode Shaw modifiée telle que nous l'avons définie (Le Lait, novembre 1933, p. 1081).

$3^{0}$ La matière grasse par la méthode Gerber dans les conditions d'adaptation que nous avons mises au point et publiées ( $L e$ Lait, juillet-août 1932, p. 640).

$4^{\circ}$ Les matières protéiques totales par un Kjedahl sur 2 gr. de caséine (avec $25 \mathrm{~cm}^{3}$ d'acide sulfurique, $10 \mathrm{gr}$. de sulfate de potasse pour élever le point d'ébullition de l'acide, $1 \mathrm{gr}$. 5 de sulfate de cuivre crist. comme catalyseur, ébullition prolongée une demi-heure après la décoloration complète, et distillation par la méthode habituelle en présence d'un grand excès de soude et d'un peu de zinc à l'appareil d'Aubin). Coefficient de transformation de l'azote en matières protéiques : 6,39.

$5^{\circ}$ L'acidité totale par dissolution complète de $1 \mathrm{gr}$. de caséine dans $20 \mathrm{~cm}^{3}$ de soude $\mathrm{N} / 10$ et titrage de l'excès d'alcalinité en retour en présence de phénolphtaléine.

60 L'acidité que nous appelons "extérieure " par titrage de l'acidité abandonnée par la caséine à l'eau après 24 heures de contact.

$7^{\circ}$ Accessoirement : le $p H$ électrométrique de la caséine, la détermination du fer par la méthode que nous avons mise au point (Le Lait, décembre 1931, p. 1038), la solubilité par la méthode au borax, etc.

Done, il nous est arrivé de rencontrer des caséines qui, donnant d'excellents résultats à la suite de cette série d'épreuves, s'avéraient incapables de convenir pour certaines applications industrielles, alors que d'autres produits présentant exactement la même composition, mais provenant d'une autre origine ou d'une autre époque, convenaient parfaitement.

Nous avons acquis cette conviction que J'examen des caséines en vue d'applications industrielles, devrait être basé sur les principes suivants :

A. Etude des propriétés physiques des solutions de caséines.

Pour que cette étude soit fertile en conclusions pratiques, il faudrait :

a) que les techniques d'étude des propriétés physiques des solutions de caséines soient précisées, standardisées et appliquées à des milliers d'échantillons déclarés bons, médiocres, mauvais pour tẹlle ou telle application, afin de rassembler une documentation importante qui manque ;

b) que chaque industrie précise les limites hors desquelles telle propriété ou tel groupe de propriétés ne devraient jamais sortir pour 
un usage déterminé - compte tenu de l'étude systématique préalable dont nous venons de parler.

Notons bien que cette étude purement physique fera constater un état de fait très précieux pour l'industriel qui utilise la caséine (puisqu'elle permettrait de choisir entre divers produits). Mais tout utile qu'elle est, cette étude serait insuffisante, car, d'une part, elle ne renseignera pas le producteur de caséine sur les raisons qui ont communiqué à ses produits tel ou tel défaut (et par conséquent ne lui donnera pas le moyen d'y remédier), et, d'autre part, parce que, même dans l'application industrielle, les propriétés physiques des caséines ne sont pas tout : certains accidents ont pu être rattachés à des causes purement chimiques pour des produits ne présentant pas d'anomalies physiques (industrie du papier par exemple).

C'est pourquoi nous envisageons"ce second principe :

B. Etude de la composition chimique des caséines sur un plan élargi.

L'étude ordinaire avec dosage de l'eau, des matières minérales des matières grasses, des protéines totales, de l'acidité, etc., reste et restera toujours nécessaire pour éliminer les produits franchement mauvais et renseigner le producteur sur les accidents de fabrication les plus graves et les plus faciles à éviter.

A cette étude nous proposons d'ajouter un examen complémentaire dont nous définirons ci-après les données, et qui aura pour but, non seulement de permettre la sélection entre diverses caséines déclarées identiquement bonnes par analyse courante, mais aussi et surtout de définir la naturé des différences ainsi découvertes, leur cause et par là même leurs remèdes, applicables à la fabrication chez le producteur de caséines.

S'il est bon de penser à sélectionner à posteriori les produits fabriqués au moment de leur utilisation, il est bien préférable de sélectionner à la production même de la caséine par la connaissance et l'élimination des causes d'écheos, que l'étude des propriétés physiques, et l'analyse chimique habituelle ne permettent de découvrir que dans un nombre de cas fort limité.

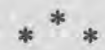

Après avoir posé et défini le problème dans son ensemble, indiquons ce que nous entendons par l'analyse chimique des caséines " sur un plan élargi ».

Que se propose l'industrie de la caséine lactique ? La fabrication de la caséine pure, c'est-à-dire d'un groupe de protéines ayant des propriétés assez bien définies (insolubilité dans l'eau par exemple) et 
ne renfermant comme substances constitutionnelles que des métalloïdes (phosphore, soufre) et aucun métal.

On connait les conditions qui permettent d'obtenir cette caséine théorique - ou du moins de s'en rapprocher autant que possible. Les nombreuses recherches du Professeur PoRCHER, celles de Mile BRIGANDo ont fait faire un grand pas à cette étude. Nos recherches personnelles, toute récentes (Le Lait, janvier 1934), y ont également contribué.

On peut en déduire, à priori, les circonstances qui pourraient être l'occasion d'une faute de fabrication.

Ces circonstances sont nombreuses. Elles peuvent se grouper en :

$1^{0}$ Circonstances intéressant le lait (état bactériologique, acidité).

$2^{\circ}$ Circonstances intéressant la fermentation lactique (température, etc.).

$3^{\circ}$ Circonstances intéressant la caillebotte (cuisson, lavage, pressages, nouvelles fermentations éventuelles).

$4^{\circ}$ Circonstances intéressant la caséine (mode 'de séchage et de conservation).

Quand on examine arec soin chacun de ces stades de la fabrication de la caséine lactique, on s'aperçoit que,les conditions de travail théorique sont celles qui consistent :

a) A ne produire aucune protéine dégradée à partir de la caséine initiale. (Cette condition intéresse l'état bactériologique du lait, certains aspects de la fermentation lactique, et surtout la caillebotte fabriquée, qui, avant séchage, ne doit être le siège d'aucune fermentation ni d'aucune hydrolyse.)

b) A ne fixer sur la molécule protéique aucun élément qui n'y soit lié naturellement. Autrement dit, il faut produire la caséine sans la faire réagir avee le milieu où elle a pris naissance. Est-ce à dire qu'il faille toujours et nécessairement effectuer la précipitation au point isoélectrique ? Pas nécessairement. Nous y reviendrons.

De toute façon, il ne faut, la caséine une fois terminée et lavée, ni caséinates, ni sels de caséine. (Cette condition intéresse l'acidité du lait, la température de précipitation, la cuisson et même le lavage de la caillebotte.)

c) A ne laisser en mélange avec la caséine produite aucune substance organique ou minérale ; d'abord, parce que, à priori, on n'aurait pas de la caséine pure, et ensuite parce que ces substances mélangées, sous l'influence de certains traitements industriels, pourraient entrer en combinaison pour fausser les propriétés physiques ou mécaniques du produit. (Cette condition intéresse surtout le lavage et le pressage de la caillebotte.) 
L'analyse chimique ordinaire, telle que nous l'avons définie précédemment, ne renseigne pas sur le premier de ces points. Elle dose l'azote total. Elle multiplie ce taux d'azote par un coefficient unique $(6,39)$, qui a été reconnu exact pour la caséine théorique, mais qui est évidemment faux pour les polypeptides de dégradation, pour les dicétopipérazines constamment rencontrées dans l'hydrolyse des protéines et à plus forte raison pour les acides aminés qui peuvent également se former sous l'influence de certains ferments à partir des formes desmotropes des cyclopeptides de la caséine.

L'analyse chimique ordinaire est en outre incapable de distinguer entre les éléments liés anormalement à la molécule protéique et les éléments mélangés à la caséine dans un produit bien précipité mais mal lavé. On dira que cette distinction a peu d'importance puisque dans les deux cas il s'agit de déterminer un élément étranger à la easéine proprement dite et donc, indésirable. Nous pensons, bien au contraire, que cette distinction est capitale, car un élément fixé chimiquement à la caséine et impossible à enlever par lavage (done pouvant exister dans un produit bien lavé, mais mal précipité) n'a pas la même signification pour le fabricant de caséine, que le même élément simplement mélangé physiquement et non combiné chimiquement, c'est-à-dire pouvant correspondre à un produit mal lavé, mais bien précipité. Les conclusions pratiques ne seront pas les mêmes. La rectification d'une fabrication dévoyée ne se fera pas de la même façon et cela, l'analyse chimique telle qu'on la fait ordinairement et l'analyse physique du produit sont incapables de le dire.

S'il est exact (et nous le pensons jusqu'à plus ample informé) que tous les accidents justiciables de chacun des stades de la fabrication d'une caséine lactique puissent se ramener en dernière analyse ì la présence dans le produit fabriqué :

a) do protéines dégradées,

b) d'éléments étrangers minéraux ou organiques fixés chimiquement à la caséine,

c) d'éléments étrangers minéraux ou organiques, mélangés physiquement à la caséine,

on est fondé à conclure que le principe d'une méthode complémentaire d'analyse intéressante doit être le suivant : étude de la composition chimique de la macération aqueuse d'une caséine," dans certaines conditions précises.

En effet :

La caséine pure est insoluble dans l'eau. La majorité de ses produits normaux d'hydrolyse (diastasique ou fermentaire) sont solubles dans l'eau. Cette forme d'altération des caséines lactiques pourra donc s'étudier (sous certaines réserves) par l'examen des solutions aqueuses de leurs macérations. 
Les éléments minéraux ou organiques simplement mélangés à la caséine et y subsistant par suite de mauvais lavages sont tous plus ou moins solubles dans l'eau (exemple : lactose, acide lactique, lactates, phosphates, chaux, etc.). La mise en solution sera plus ou moins aisée suivant les cas.

Les éléments minéraux ou organiques fixés éventuellement sur la molécule de caséine, ne peuvent généralement pas être mis en solution dans l'eau par macération pure et simple. L'analyse chimique détaillée de la caséine totale ou de solutions sodiques de la caséine pourra renseigner utilement par comparaison avec les résultats de l'examen des macérations aqueuses.

Dans tous les cas, eet examen d'ensemble devra comporter le dosage d'un eertain nombre d'éléments minéraux ou organiques dans des macérations aqueuses, dans les solutions sodiques ou dans la caséine elle-même. Dans chaque eas, nous nous efforcerons :

10 de fixer la technique du dosage;

$2^{\circ}$ de l'appliquer à des caséines préparées de manière telle que les principaux accidents de fabrication aient pu être reproduits ;

$3^{\circ}$ d'en tirer, comme conclusion pratique, l'enseignement qu'à telle anomalie analytique, découverte par les móthodes qui seront données, correspond une anomalie de fabrication à laquelle il peut être, dans chaque cas, porté remède.

Le couronnement de cette étude (qu'il nous sera impossible de réaliser) devrait être l'examen des accidents constatés lors de l'utilisation des caséines et le rapport qui existe entre ces accidents et certains aspects de la composition de ce produit, pour pouvoir ensuite remonter aux accidents de fabrication de la caséine.

Nous ne pourrons donc pas dire : tel accident observé dans l'industrie du papier correspond à telle anomalie de constitution et, de là, à telle anomalie de la préparation de la caséine. Cette étude complète nécessiterait une collaboration étroite avec les diverses industries qui utilisent ce produit. Mais nous formons l'espoir d'être amené, dans une certaine mesure, à pouvoir dire : telle caséine qui paraissait bonne au producteur et au client, présente une anomalie de constitution et cette anomalie provient de telle circonstance de sa fabrication, à laquelle il convient d'apporter telle modification.

\section{En résumé :}

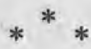

$1^{0}$ Il existe des caséines qui, paraissant bonnes à la suite d'un examen chimique ordinaire, sont cause d'accidents constatés lors de leur utilisation dans certaines industries. 
De même, des caséines d'égale composition se comportent parfois différemment dans une même industrie.

$2^{\circ}$ L'examen chimique habituel nous laisse done ignorer dans la composition des caséines, certains aspects générateurs " d'accidents d'utilisation ".

$3^{\circ}$ L'étude physique des caséines permettrait dans quelques cas de prévoir certains accidents d'utilisation. Mais les accidents afférents à un aspect chimique de la caséine resteraient imprévus et dans tous les cas on serait dans l'ignorance à peu près complète du lien qui unit ces anomalies de composition, aux anomalies correspondantes dans la fabrication de la caséine et des remèdes à y apporter.

$4^{\circ}$ L'étude de ces questions permet de penser que l'analyse chimique des macérations aqueuses et des solutions de caséine serait de nature à renseigner sur des aspects de la composition que l'analyse ordinaire ne permet pas de découvrir.

$5^{\circ}$ C'est cette étude que nous exposerons en donnant, d'une part les techniques d'examen, d'autre part l'interprétation à donner aux résultats obtenus, e'est-à-dire, la possibilité, connaissant les anomalies de composition, d'en déduire les anomalies de fabrication de la caséine et les remèdes qu'elles comportent.

(A suivre.)

\section{BIBLIOGRAPHIE ANALYTIQUE}

\section{LES LIVRES}

\section{Dresrousses (Lucienne). - La dyspepsie du lait de femme.}

Thèse Doct. Méd., Paris. Une brochure de 112 pages. Jouve et $\mathrm{C}^{\mathrm{ie}}$, éd., 15, rue Racine, Paris, 1930.

L'enfant au sein n'est pas absolument à l'abri des troubles digestifs, mais ils restent chez lui habituellement bénins. Il est exceptionnel que par leur intensité, leur ténacité, ils entraînent des troubles sérieux de la croissance qui nécessitent la suppression d'un tel allaitement. Dans la plupart des eas on arrive à faire tolérer le lait maternel.

Les troubles digestifs du nourrisson viennent quelquefois d'une composition anormale du lait, et l'analyse faite dans de bonnes conditions peut rendre d'utiles services.

C'est la richesse spéciale du lait de femme en lactose qui donne à la diarrhée du nourrisson au sein sa physionomie particulière.

Les dyspepsies tenaces s'observent surtout chez les enfants prédisposés par leur héridité : neuro-arthritique, syphilitique, accessoirement alcoolique ou tuberculeuse.

Parfois, c'est dès la naissance que l'enfant est intolérant au lait de femme. Ch. PORCHER, 